\title{
The Application of Entertainment Design And Digital Technology in Children's Educational Exhibition Space-Take the "Art Education in Fun" exhibition as an example
}

\author{
Cheng Si-hang ${ }^{1,}$, , Wang Yun-long ${ }^{1, b}$ \\ ${ }^{1}$ Dept: School of Design, Jianghan University, Wuhan, Hubei, China
}

\begin{abstract}
The purpose is by integrating entertainment design and digital technology thinking into the display space design, enhancing spatial narrative and interactive experience, stimulating children's interest and imagination, and cultivating innovative thinking mode. The methods is taking the exhibition planning of "Art Education in Fun" as an example, visually displaying the narrative space, combined with digital technology and entertainment interaction, strengthening the fit between the two-dimensional plane and the threedimensional space, and providing the audience with a more dimensional and stronger textured exhibition experience. The conclusion is with the addition of entertainment design and digital technology, deepening the basic value of the information dissemination in the exhibition space itself, building a better information exchange platform, and providing a positive boost to culture, education, and industrial economy.
\end{abstract}

\section{Introduction}

At present, most children's art education spaces continue the single linear teaching mode in the classroom, neglecting the cultivation of the children's creative thinking, and leading to the transformation of art education into pure art education. And this kind of creative thinking plays a very important role in the growth of children, especially in the cultivation of logical thinking ability. The "Art Education in Fun" exhibition adjusts the space environment accordingly to children's cognition level, and adds entertainment design, from space narration, visual design and digital entertainment design, make the interaction between children and the space reaching an ideal state and presents the display space more scientific.

\section{Space Narrative}

The narrative is a kind of literary carrier, which aims to bring the audience a complete combination of stories through abstract concepts and enhance the sense of substitution. On this basis, space narration strengthens the dialogue in the space, and enhances the authenticity of the story through the design of basic elements such as color, lighting, sound effects, senses and exhibits. At the same time, the audience can quickly get the sense of substitution in to the exhibition, enhancing the immersive experience, and organically combining the relationship between space, exhibits and people, creating more imagination space, and then leaving a profound exhibition experience. According to research on child psychology, children have a higher tendency to bright and warm colors, so the "Art Education in Fun" exhibition is mainly in warm orange tones, using natural light and indoor light to create a bright and warm space environment (figure 1). The digital interactive design of the exhibition uses embedded speakers to cooperate with the interactive content for sound transmission effect to make the exhibition more vivid and enhance the narrative of the space. By stimulating sense of sight, hearing, touch and smell, the audience's mood are fully mobilized, the sense of reality and experience in narrative space is enhanced, and the impression of exhibitors is deepened.

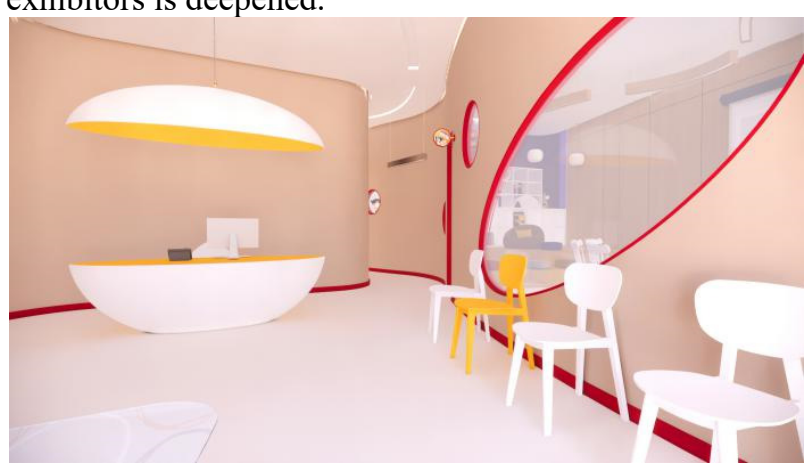

figure 1-space environment

Traditional educational spaces are mostly presented in the form of square boxes with a single form, ignoring the benign interaction between the creation of the space atmosphere and the cognitive development of children, and the space environment plays an indispensable role in the development of children's psychological and logical thinking. With the development of digital technology, traditional culture education has gradually turned to digital culture education. Art education space should break this 
kind of solidification mode, and create an environment that can attract children's attention and mobilize learning enthusiasm. By strengthening the space narrative, the space is no longer a cold teaching place, but a flexible space for dialogue with children and assisting the development of educational work. Taking the exhibition hall design as an example, the exhibition channel is cut through the circle to make the channel winding and round, arousing the desire of the audience to explore and visit (figure II). The sub-exhibition hall also avoids the appearance of rigid right angles, and is mainly based on arcs, giving the exhibition hall a gentle attribute, which is more acceptable to children, so as to quickly integrate into the exhibition environment. The display of the content carries the specific language form of the narrative space, but also strengthens the interaction between people and objects. In the "Art Education in Joy" exhibition, the narrative and story of space are strengthened through diagram annotation, illustration and related background, so that the audience can better understand the exhibits.

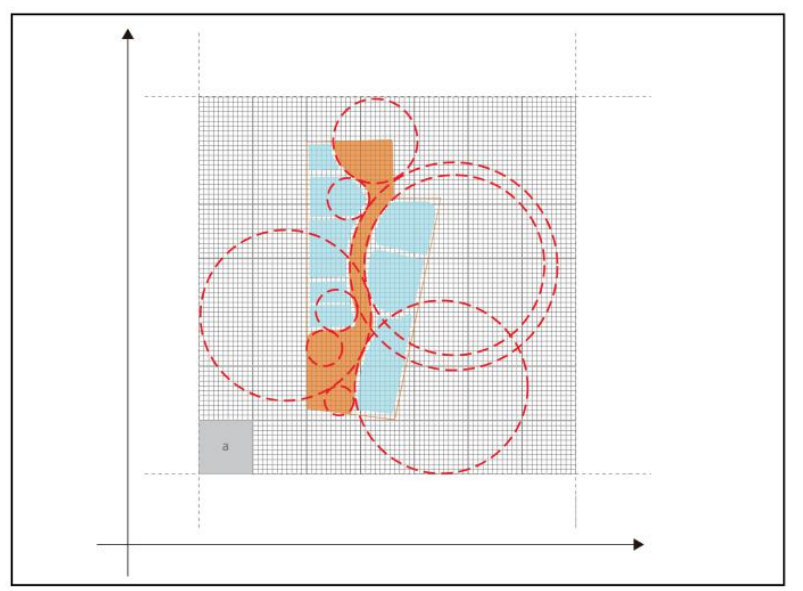

figure II-channel

Modern exhibitions are mostly designed from the perspective of adults and with adult mindsets, with space language being too solemn, which hardly arouse children's interest. The "Art Education for Fun" exhibition utilizes double rows of small space combinations as a means, by which a warm, comfortable and secure space is created. Through the decorative design of walls and floors, it breaks through the sense of distance in the traditional exhibition space while making the exhibition space compatible with the characteristics of children's physical and mental development. Simultaneously, family experience rooms are provided to enhance the interaction between parents and children, strengthen the level of exhibition and deepen the long-term memory brought by the exhibition experience. With the creation of spatial environment, space narrative is enhanced, facilitating the interaction between people, exhibits, and space, so as to establish the audience's own experience story. There is a bi-directional function between exhibit display and spatial narrative, which creates the background and atmosphere for the exhibits. The exhibit display contributes to the figurative expression of the spatial narrative, both of which complement each other to jointly deliver the exhibition theme.

The narrative design of space is not only a design method, but also a mode of thinking ${ }^{[1]}$. Aiming at the development of children's psychological cognition, metaphors, symbols, exaggerations and other rhetorical techniques are used in spatial narration to attract children's interest and guide children to actively participate in the exhibition. Through personal experience and interaction with the exhibition environment, education can improve children's cognitive structure in a benign interactive way. At the same time, we enhance the audience's immersive experience by the application of scenes, different perspectives, the first-person instead of the third-person, digital technology and scene reappearance. The exhibition "Art Education in joy" incorporates a large number of innovative digital technologies, such as AI, VR experience and AR technology, to visualize and materialize knowledge, which enhances the educational attributes (figure III). The "Art Education in Fun" exhibition takes art training as an entry point, amplifies children's imagination, cultivates creative thinking, and provides an innovative thinking for a commercial education space that tends to be homogeneous.

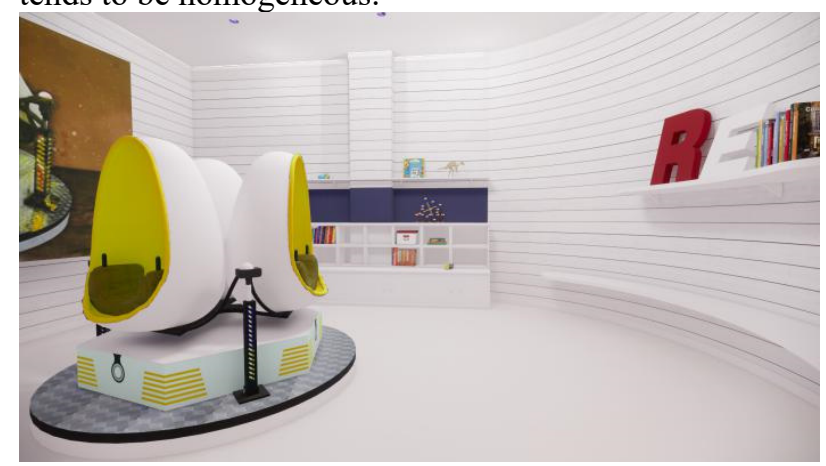

figure III-VR experience

\section{3 visual presentation}

In the content design of children's exhibition space, designers need to stand in the perspective of children, transform the text into vivid design language and express $\mathrm{it}^{[2]}$. Visualizing the narrative space helps children understand the abstract spatial concepts which make children be easier to resonate with the content of the exhibition. And to the space design, there must be first with the design concept, and then the space structure, we cannot turn the cart before the horse. Through the analysis of children's value scale, behavior habits, psychological cognition, and the ontological research of the environment, atmosphere and function of the space, the design concept is determined. By formulation of the design concept, the mood board and the story board, the coordination with the design trend of the exhibition, achieving the matching of the space structure and the display content, making the exhibition more scientific. By setting the script of the exhibition, the exhibition order, exhibition hall function and emotion mobilization are organically designed, and the addition of digital technology optimizes the user experience (figure IV). 


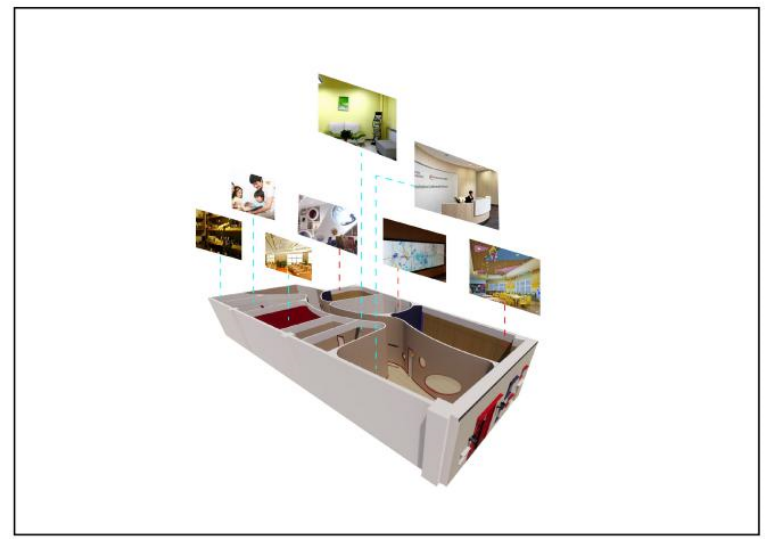

figure IV-functional partitions

Vision is an intuitive image that is first felt through the physiological senses, and brightly colored visual images are more likely to arouse children's interest. The plane system that constitutes the two-dimensional space can assist the conceptual expression of the three-dimensional space, and at the same time, its own propaganda and promotion attributes can create momentum for the exhibition. Taking the "Art Education in Fun" exhibition as an example, the main visual of the promotion adopts $2.5 \mathrm{D}$ axis-side isometric illustrations to show in detail the functional partitions of the exhibition hall, the audience can form a certain spatial concept of the exhibition. Arouse interest in the exhibition, Compared with the daily seen flat posters, posters with a stronger three-dimensional effect and rich colors are also easier to attract children's attention (figure V). By repeatedly presenting a large number of visual images, a visual exposure effect is formed, and audience memory is enhanced, which is of great benefit to the conveying of the exhibition concept. As a part of the exhibition space, the exhibition visualization also belongs to the exhibits, which runs through the entire exhibition and is the backbone of the entire story line, ensuring the unity and rhythm of the exhibition.

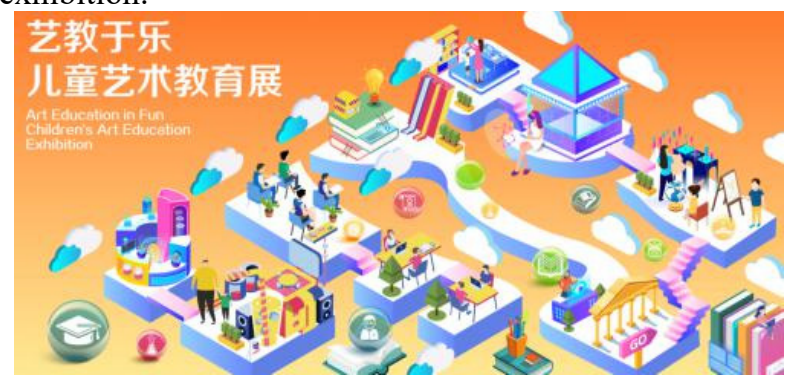

figure $\mathbf{V}$-main visual

In addition to the publicity of the main vision, the color and graphic design of the logo, mascot, publicity materials, and guidance system must also be determined to form a complete visual publicity image. Pay attention to the physical and psychological feelings brought to the audience by the brand image and exhibition space, enhance the emotional and interesting experience, and achieve the ideal state of resonance between the audience and the exhibition. Especially when the audience of the exhibition is children, we should consider whether the visual system of the exhibition will attract children's interest, whether it will cause visual disgust, and whether it can be interpreted by children. But at the same time, attention should be paid to avoiding the clutter of the visual system style caused by the increase of promotional materials, it is necessary to unify the visual image, enhance the signal-to-noise ratio, and reduce some unnecessary "noise" to achieve the ideal state. At the end of the exhibition hall, the "Art Education in Joy " exhibition features cultural and creative products as an extension of the main visual and mascot, which is given away free of charge as a souvenir of the exhibition, bringing positive impact to the exhibition, enhancing the brand image and awareness. Focusing on the visual image of the exhibition, exploiting the timeliness and topicality of the exhibition, depending on the self-media platform for promotion, and the secondary forwarding by visitors, sustainable communication and promotion can be generated for the exhibition.

\section{Digital Entertainment}

The core of digital entertainment is the game design aimed at the psychology of curiosity, which provides virtual entertaining user experience through participation and interaction. Digital entertainment design has the characteristics of literary and artistic creative forms, multimedia and networked interactive expression methods, and cross-integration of multidisciplinary fields [3]. Therefore, digital entertainment assists the visualization of space narratives from the aspects of culture, art, technology, etc., injects emotional factors into the space, and deepens the connotation and texture of the exhibition. The proportion of digital entertainment design in the education field is gradually increasing, and it has become a new trend in digital education in terms of knowledge visualization, artistic perception, and thinking creativity. The development of interactive multimedia is becoming more and more perfect, aiming to the audience and theme of the exhibition, with interactive design of different forms and contents, as well as barrier-free use, enhance the audience's exhibition experience and aesthetic enjoyment.

The introduction of digital technology in the exhibition compensates for the limitations in information transmission and educational properties of the physical exhibition, integrating the concept of "good storytelling" throughout the exhibition with the combination of reality and imagination. Digital technology integrates the stimulation of the senses in the narrative space, stimulating one sense to trigger another, thereby delivering a more multi-layered experience to audiences, enhancing entertainment while simultaneously reserving more space for imagination. There is a section entitled "Hearing Colors" in the "Art Education for Fun" exhibition. With headphones on, children are able to draw and color pictures by judging the types of audible objects according to some sounds extracted from nature being played in the headphones. It stimulates children's imagination and color perception, presents visual images in their minds, and exercises logical thinking skills.

The modern exhibition industry has long been not 
limited to physical exhibitions, combined with virtual digital media, to break the shackles of space and time in traditional exhibitions, and enhance the entertainment, interaction and user experience of the exhibition. As a cross-border digital technology, it can bring visitors a fresh sense of multidimensional visit and provide digital representation for scenes that are difficult to construct. Virtual reality technology is a simulation of the real environment, which realizes the transmission of information and the perception of the situation through the experience of the environment ${ }^{[4]}$. The exhibition "Art Education in Fun" divides the exhibition hall into six themed units, and the flow of the exhibition is based on the order of hierarchy of artistic creation, through the intervention of new media such as digital entertainment, the connection between each unit is strengthened and the exhibition theme is set off together. Taking the exhibition creation room as an example, the works created by children on the spot can be scanned and imaged by a scanner, which can be projected into their corresponding scenes, so that the created works can be moved and the organic combination of popular science education and art can be realized. Somatosensory interaction is added in the picture book room, and children can use gestures to control the reading in front of the screen, which enhances the interest of reading (figure VI).

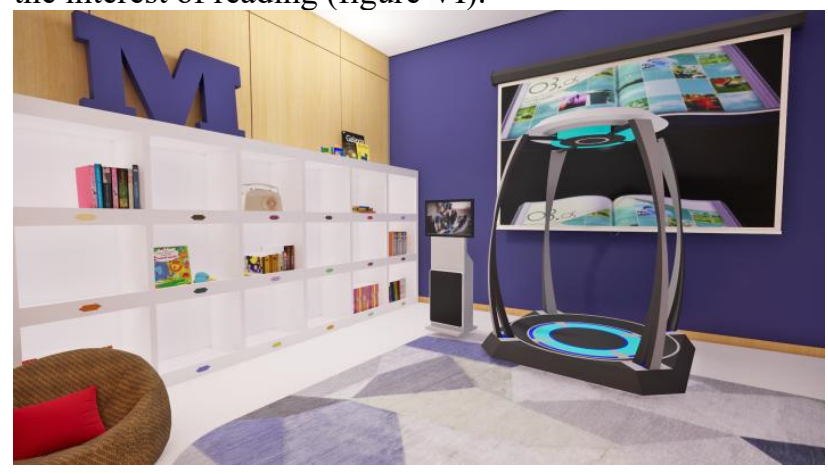

figure VI- picture book room

The interaction between space and people forms a four-dimensional space with different perspectives, which strengthens the emotional communication between the environment and people, and facilitates the communication of the exhibition concept. According to the research on children's mental models, the "Art Education in Fun" exhibition uses the learning, entertaining, and interactivity of interactive displays to explore the exercise of children's operation ability, autonomous learning ability, and artistic perception, the experience is different from the classroom which cultivates creative thinking. Conventional classrooms are based on indoctrination learning methods, which put children in a passive position and are prone to boring emotions. The "Art Education in Fun" exhibition reproduces the scene through digital entertainment interactive technology, children substitute themselves into the scene through their own creations, and use this as a way to display popular science and expand their thinking, arousing a strong thirst for knowledge and leading a positive learning attitude. The exhibition also features an art-related crossword puzzle game, in which children who solve the puzzle will be given limited edition gifts of cultural and creative products after the visit and information gathering (figure VII).

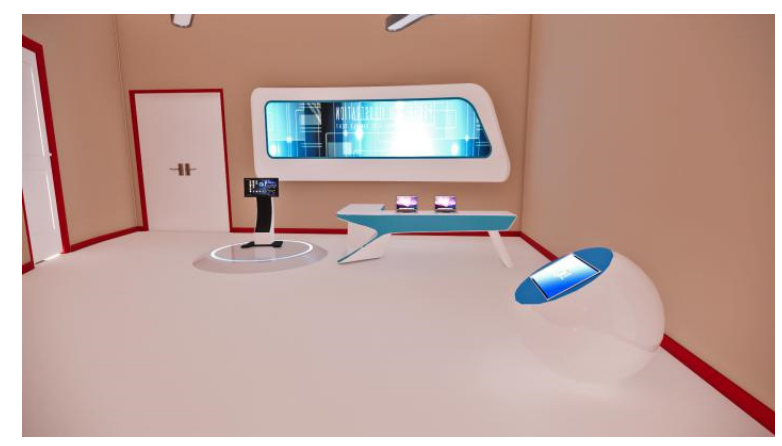

figure VII- Puzzle table

\section{5 conclusion}

Children's educational space should be given more attention, and should not only focus on the appearance design of simple cartoons, but on children's emotional factors. The new digital culture format has been gradually accepted by most people. It is an effective way to drive the development of cultural industry and commercial industry through innovative digital technology under the new cultural background. Business education space has both cultural and economic attributes, while the innovation, cross-border and cultural nature of digital technology can bring added value to the exhibition and meet the needs of audience. The "Art Education in Fun" exhibition has made a new direction of practice for the various but single art education in the market, it is also a study of the relationship between space environment and education from the perspective of children, the design is not only for serving one kind of crowd, but for humanized design for different ages, classes and levels of needs. By adding entertainment design, it adds fun and entertainment to the exhibition, and at the same time adds more cultural and artistic value to marketing activities with economic attributes. Digital entertainment design has gradually matured in our country, and I hope that it will collide with display design to create more sparks.

\section{References}

1. Yang Yan. On the narrative design of space[J]. Hundred Schools In Art, 2007

2. Yang Qi. The experiential situational design of children's science exhibition hall —-Taking Huangshan Environmental Education Base as an example [J]. Decoration, 2018

3. Fu Zhi-yong. The positioning of digital entertainment design and the training of compound talents[J].Decoration, 2007

4. Ren Li-fen. Application analysis of virtual reality technology in modern display and communication[J].Publishing Wide Angle,2019 